\title{
Holographic description of color superconductivity
}

\author{
Kazem Bitaghsir Fadafan, ${ }^{1}$ Jesus Cruz Rojas, ${ }^{2}$ and Nick Evans ${ }^{2}$ \\ ${ }^{1}$ Faculty of Physics, Shahrood University of Technology, P.O. Box 3619995161 Shahrood, Iran \\ ${ }^{2}$ STAG Research Centre \& Physics and Astronomy, University of Southampton, Southampton, \\ England SO17 1BJ, United Kingdom
}

(Received 19 March 2018; published 18 September 2018)

\begin{abstract}
The difficulty of describing the gauge dependent biquark condensate in the QCD color superconducting phase has made it hard to construct a holographic dual of the state. To side step this problem, we argue that near the chiral restoration transition in the temperature-chemical potential plane, the strongly coupled gluons are likely completely gapped so that the color quantum numbers of the quarks can be thought of below that gap as global indices. A standard anti-de Sitter-superconductor model can then be used to analyze the fermionic gap formation. We investigate the role of four-fermion interactions, which might be used to include the gapped QCD interactions, on the vacuum and metastable vacua of the model. It turns out to be easiest to simply relate the standard interaction of the holographic superconductor to the strength of the gapped gluons. The result is a holographic description of the QCD color superconducting phase diagram. We take a first look at how quark mass enters and causes a transition between the color-flavor locked phase and the two flavor color superconducting phase.
\end{abstract}

DOI: 10.1103/PhysRevD.98.066010

\section{INTRODUCTION}

A fermionic system at finite chemical potential is expected to develop a Fermi surface. It is known that if there is any attractive interaction between the fermions, Cooper pair condensation will occur, causing superconductivity or superfluidity. This was made transparent to a particle physics audience by the renormalization group flow analysis of Refs. [1] (updated to a relativistic system in Ref. [2]). This fact leads to the natural expectation that quarks will condense in high density QCD, and there has been considerable work on understanding the phase structure over a number of years (see, e.g., the review [3]). Typically, the preferred condensation channel is expected to break the color gauge group, so the phenomena is referred to as color superconductivity (CSC).

At very large chemical potentials, QCD is believed to become weakly coupled due to asymptotic freedom, and an exact computation of the condensation pattern is possible [4]. The more experimentally interesting case, though, is when the density and temperature of the quark gluon plasma are of order the strong coupling scale $\Lambda_{c}$, and here the strongly coupled nature of the problem makes precise computation tricky. Gap equation and renormalization

Published by the American Physical Society under the terms of the Creative Commons Attribution 4.0 International license. Further distribution of this work must maintain attribution to the author(s) and the published article's title, journal citation, and DOI. Funded by SCOAP ${ }^{3}$. group analysis have been done, and there is a large amount of literature on the possible phase structure as a function of $N_{f}$ and the quark masses [3].

Over the last two decades, holography has emerged as a new tool to study strongly coupled gauge theories [5]. It provides the ability to rigorously compute in theories close to large $N_{c} \mathcal{N}=4$ super Yang-Mills theory (including theories with quarks [6]) using a weakly coupled gravitational/stringy dual. The framework has been expanded phenomenologically to anti-de Sitter (AdS)/QCD type models of a wider space of theories [7]. It has been natural throughout this period to attempt to study the CSC phase of QCD with this new tool. There is immediately a number of large obstacles, though. The CSC effect is subleading in the large $N_{c}$ limit [8]. The condensate depends on $N_{c}$, so there is no clear large $N_{c}$ limit. Finally, the dimension-3 condensate likely breaks the gauge group, yet on the gravitational side, only gauge invariant operators are manifest, so it is not clear how to even pose the problem (the gauge invariant square of the operator is dimension 6 but a stringy state in the dual theory). Nevertheless, an instability to pair condensation of gauginos, which can form a color singlet pair, in the presence of a chemical potential was observed, e.g., early on in Ref. [9]. This idea was phenomenologically used to develop AdS descriptions of superconducting condensed matter systems [10], leading to the AdS/Condensed Matter (CM) field of study. Holographic studies of related instabilities in theories with scalar quarks have also been studied in Refs. [11].

In this paper, we want to return to the problem in QCD. The obstacles above remain grave, so our approach will be to side step them. In the intermediate density phase of 
QCD, the quark gluon plasma is strongly coupled and full of free electrically charged quarks and presumably composite, magnetically charged scalars (see Ref. [12] for a recent discussion). The latter condense below the chiral phase transition to cause confinement (at least in the pure glue theory). Above the transition, such states will still be present if not condensed. The expectation is that these fields, through loop diagrams, will generate a Debye mass of order $g \sqrt{T^{2}+\mu^{2}}$ for both the electric and magnetic gluons (the latter are not gapped at weak coupling where there are no magnetic charges present [13]). We will posit here that, because $g$ is large, there can be an order of magnitude gap between the gluon mass and the chemical potential/temperature scale. We will squeeze a holographic description, in the spirit of AdS/QCD, into this energy regime. Since the gluons are gapped, we will dodge the issue of treating the $\mathrm{SU}(3)$ color symmetry of the quarks as a gauge symmetry and instead impose it as just a flavor symmetry. Although the biquark condensate will further gap the gluons, we presume this to be a small effect relative to the Debye screening. These assumptions will save us from the problems encountered holographically to date.

We stress that we work in a phenomenological bottomup fashion in the spirit of AdS/QCD (at $N_{c}=3$ ) or AdS/ $\mathrm{CM}$ (where phonon interactions of electrons are described). We will use an AdS space to phenomenologically describe the conformal symmetries of the free fermions below the Debye gap scale, which are then broken by the operators and sources of the theory that appear in the bulk-for example, temperature, chemical potential, etc. One should of course worry that at low $N_{c}$ the bulk modes might become strongly coupled and stringy, but the AdS/QCD philosophy is to soldier on and measure success by the output. Our model is at heart the simplest AdS/superconductor model [10]. We still need to correctly describe the broken QCD interactions that generate the Cooper pair condensation. We will discuss reintroducing the interactions as four fermion terms using Witten's double trace prescription [14] (recent work on developing the holography of four fermion operators can be found in Refs. $[15,16])$. There are subtleties in this analysis including excited states of the vacuum, and it turns out that an infinitely repulsive force is needed to switch off the inherent attractive channel of the base AdS/superconductivity model. We will conclude we should just choose to tune the intrinsic pairing interaction of the holographic model to represent the broken QCD interactions on the global color degrees of freedom (d.o.f.). The goal of this Paper is to study such broken gauge interactions in the quark gluon plasma to develop a sensible description of the CSC phase in the QCD phase diagram.

Let us quickly review the CSC condensation patterns that will interest us here [3]. The superconducting condensation is triggered by a chemical potential for $\mathrm{U}(1)_{B}$ and the associated quark number density. In all cases, we are interested in the condensation of a biquark operator with quark number 2 or baryon number $2 / 3$. We assume that at strong coupling the $\overline{3}$ color channel remains attractive as at weak coupling while the 6 is repulsive so the condensation is the usual antisymmetric $\overline{3}$ state. A spin 0 condensate is formed from an antisymmetric combination of spins. The flavor wave function of the condensate must also therefore be antisymmetric. First, with three massless quark flavors, this implies the condensate is an antisymmetric flavor $\overline{3}$ also. We can represent this state by the matrix (we show the makeup of the $\overline{3} \mathrm{~s}$ of color and flavor in terms of the constituents)

\begin{tabular}{|c|c|c|c|c|}
\hline & $\bar{R}$ & $\bar{G}$ & $\bar{B}$ \\
\hline & & $B G-G B$ & $B R-R B$ & $R G-G R$ \\
\hline $\bar{u}$ & $s d-d s$ & $\Delta_{1}$ & & \\
\hline $\bar{d}$ & $s u-u s$ & & $\Delta_{2}$ & \\
\hline $\bar{s}$ & $u d-d u$ & & & $\Delta_{3}$ \\
\hline
\end{tabular}

In the three flavor massless limit the expectation is that the condensate will be the diagonal as shown with all $\Delta_{i}$ equal-this is the color-flavor locked state [17]. As the strange quark becomes massive, the condensates of the top two rows $\left(\Delta_{1}, \Delta_{2}\right)$ switch off, and we expect to find a vacuum expectation value (vev) for the triplet, $\mathrm{SU}(2)$ flavor singlet of the bottom row $\left(\Delta_{3}\right)$-this is the 2SC phase of the massless two flavor case. Note all of these states carry net color charge, although we have argued the main source of gluon mass is the Debye screening rather than the Meissner induced mass. In the holographic model, we will describe an AdS-scalar $\psi$ that is dual to an element $\Delta_{i}$ of this matrix which acquires a vev. We will seek the phase boundary where the condensate switches on in the $T-\mu$ plane. We will briefly discuss including a quark mass in our final section to display a transition between the color-flavor locked and the 2SC phases, although as we will stress, the analysis is very naive, and challenges remain to find a complete holographic picture.

In Sec. II, we will review the origin of the electric and magnetic Debye gluon masses that generate a gap. In Sec. III, we review the AdS superconductor model that we will use including fields for each of the biquark gaps we consider. In Sec. IV, we look at the role of four quark operators in the supercondutor model including the role of unstable minima of the model. In Sec. V, we match the superconductor model's coupling to the QCD coupling in the $T-\mu$ plane to predict the gap size. In Sec. VA, we discuss how quark mass would enter the holographic model to suppress the biquark condensates. Finally, in Sec. VI, we conclude. 


\section{ELECTRIC AND MAGNETIC DEBYE MASSES}

Our arguments about the gapping of the gluonic d.o.f. are important to our approach, so we will briefly review the ideas already in the literature in more detail. At high density QCD is believed to become weakly coupled, and one can explicitly compute in perturbation theory [13]. Here, it is then known that the electric $A_{0}$ gluon components acquire a Debye mass of order $g \mu$. The magnetic $A_{i}$ d.o.f., though, are not fully screened but instead Landau damped. Their self-energy behaves as $\Sigma^{2} \sim g^{2} \mu^{2}\left|q_{0}\right| /|q|$ with $q$ the gluonic fourmomentum. In such weakly coupled theories, if color superconductivity sets in, then the charged gap is the only source of mass for the magnetic gluonic d.o.f. Indeed, a central point of the analysis in Ref. [4] was to include the effects of the Landau damping in the estimate for the gap scale.

We argue, though, that at low chemical potential, which is relevant for neutron star and heavy ion collisions, the behavior is probably rather different. In particular, we expect the QCD plasma to contain magnetically charged scalars (see Ref. [12] for a recent discussion) because of their role in confinement below the chiral/deconfinement transition. If such (composite) states do exist, then they will simply through one loop diagrams generate a Debye-like mass for the magnetic $A_{i}$ gluonic d.o.f., too. Now, all the gluons are gapped at the scale $g \mu$, which for chemical potentials in the hundreds of $\mathrm{MeV}$ and for $g \sim 4 \pi$ are much higher than the superconductor gap scale, which is typically estimated in the tens of MeV. This separation of scales motivates a description of color supeconductivity in which the quarks exist as the sole d.o.f. in the low energy theory below $g \mu$ interacting only by four fermion operators generated by the gluons. In such a description, the color quantum numbers of the quarks will appear as global quantum numbers (although a full description of all higher-dimension operators would secretly include gauge invariance). Since holographic color superconductor models describe the breaking of global symmetries, we can now hope to apply that framework to this energy regime in high density QCD. Note that we assume that the contribution to the gluon gap from the low scale superconducting condensate is small relative to the Debye masses generated by the plasma so that the cutoff scale and gap can be considered disconnected.

\section{ADS SUPERCONDUCTORS}

As a first start in this paper, we will just address the CSC phase, which is the novel physics of interest. We will assume the chiral transition, where the $\bar{q} q$ condensation occurs, is at the scale where the QCD coupling diverges $\Lambda_{c}$. Thus, consider $T, \mu$ scales above this energy scale only. One finds the phase diagram in Fig. 5.

Let us begin by setting up a very simple AdS description of superconductivity following the start up model of Ref. [10]. We place our description in a black hole geometry (which we will not backreact) $d s^{2}=r^{2}\left(-f d t^{2}+d \vec{x}^{2}\right)+\frac{1}{r^{2} f} d r^{2}, \quad f=1-\frac{r_{H}^{4}}{r^{4}}$.

Here, $\vec{x}=x, y, z$ are the boundary coordinates, and the radial distance is $r$ so that the boundary is located at infinity. The usual relation between temperature and the horizon position is $r_{H}=\pi T$ (we have set the AdS radius to 1 ).

In our setup we consider a scalar field, $\psi_{i}$, to represent the quark bilinear $\Delta_{i}$ from (1) (here, a component of the quark bilinear in the $\overline{3}$ of color) with baryon number $B=2 / 3$ and dimension 3 , and a gauge field associated with $\mathrm{U}(1)_{B}$ of which the $A_{t}$ component will describe the chemical potential. We use an action,

$$
\mathcal{L}=-\frac{1}{4} F^{\mu \nu} F_{\mu \nu}-\left|\partial \psi_{i}-i B A \psi_{i}\right|^{2}+3 \psi_{i}^{2}
$$

the mass is picked to be minus 3 in units of the AdS radius since this corresponds holographically via $M^{2}=\Delta(\Delta-4)$ to a dimension-3 operator. We have neglected any order $\psi^{4}$ interaction terms between different $\psi_{i}$.

The equations of motion are

$$
\psi_{i}^{\prime \prime}+\left(\frac{f^{\prime}}{f}+\frac{5}{r}\right) \psi_{i}^{\prime}+\frac{B^{2}}{r^{4} f^{2}} A_{t}^{2} \psi_{i}+\frac{3}{r^{2} f} \psi_{i}=0
$$

and

$$
A_{t}^{\prime \prime}+\frac{3}{r} A_{t}^{\prime}-\sum_{i} \frac{2 B^{2}}{r^{2} f} \psi_{i}^{2} A_{t}=0
$$

As usual, for regularity, one requires $A_{t}=0$ at the horizon, which implies from the first equation of motion that

$$
\psi_{i}^{\prime}=-\frac{3}{4 r_{H}} \psi_{i} .
$$

Note that strictly at $T=0$ we cannot assume this boundary condition and the model is not complete. We will use the model to work out the edge of the phase boundary at finite $\mathrm{T}$ and not address the $T=0$ state.

There is always a solution,

$$
\psi_{i}=0, \quad A_{t}=\mu-\frac{\mu r_{H}^{2}}{r^{2}} .
$$

There are more complex solutions that we can find numerically by shooting out from the horizon. In the UV, they take the form

$$
\psi_{i}=\frac{J_{c}}{r}+\frac{c}{r^{3}}+\cdots \quad A_{t}=\mu+\frac{d}{r^{2}}+\cdots
$$

$c$ is interpreted as the Cooper pair condensate, $\mathcal{O}=\psi \psi, J_{c}$ is the source for that operator (which carries both color and flavor indices generically), $\mu$ is the chemical potential, and 
$d$ is the density. Since there are two constraints on $\psi, \psi^{\prime}, A_{t}$, $A_{t}^{\prime}$ at the horizon, we get a two parameter family of solutions [set in the IR by $\psi\left(r_{H}\right)$ and $A_{t}^{\prime}\left(r_{H}\right)$, which we label by the values of $J_{c}$ and $\mu$, predicting $c$ and $d$ ].

For example, let us consider the case with a single $\psi_{i}$ field, which might describe $\Delta_{3}$ in the two flavor case. Note that if there is more than one identical $\psi_{i}$ then there is an effective factor of $N_{i}$ in the interaction term in (5). This can be removed by rescaling the $\psi_{i}$ by $1 / \sqrt{N_{i}}$ leaving the same equations to be solved. In practice, this means the colorflavor locked (CFL) condensates will be a factor of $\sqrt{3}$ smaller than the 2SC computations we make. Crucially, though, the phase boundaries remain at the same coupling values. For this reason, we will mainly study the $N_{i}=1$ case.

Now, we can solve (4) and (5) numercially. In Fig. 1(a) for $T=0.1$, we plot the solutions of $\psi$ (we plot $r \psi$, which asymptotes to $J_{c}$ in the UV), where in each case $A_{t}^{\prime}\left(r_{H}\right)$ has been adjusted to set $\mu=1.0$. In Fig. 1(b), we show solutions for $\mu=5.0$. At low $\mu$, there is no symmetry breaking - the only solution with $J_{c}=0$ is that with $\psi=0$ so that $c=0$. For the higher value of $\mu$, the solution that asymptotes to $J_{c}=0$ is symmetry breaking (the curve

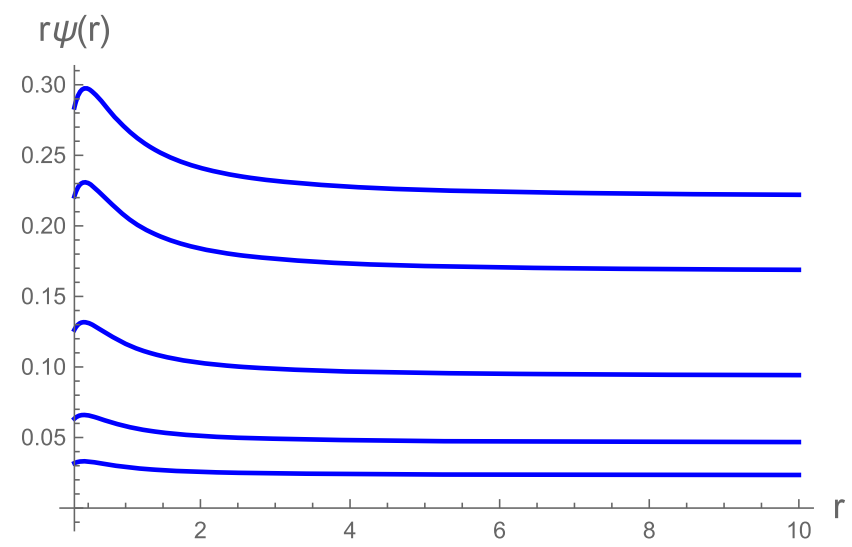

(a)



(b)

FIG. 1. (a) The $\psi$ functions in the unbroken phase at $T=0.1$, $\mu=1.0$. (b) The $\psi$ functions in the broken phase at $T=0.1$, $\mu=5.0$.

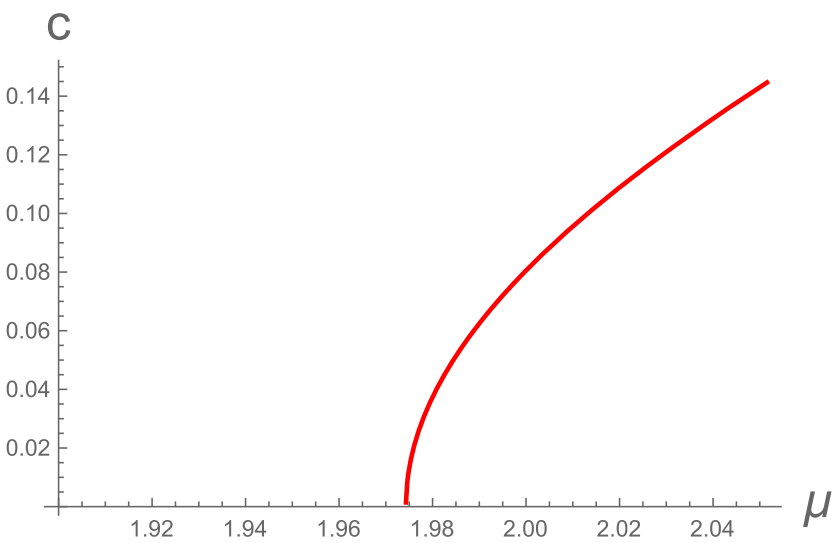

FIG. 2. The condensation vs $\mu$ in the broken phase at $T=0.1$.

shown with the highest IR value) - this solution has a nonzero condensate $c$. The physics here in AdS is that the chemical potential generates an effective negative mass squared for the scalar $\psi$, and when it violates the Brietenlohner-Freedman (BF) bound of $M^{2}=-4$ [18], an instability to $\psi$ condensation results.

In Fig. 2, we plot the value of the condensate against $\mu$ for the $J_{c}=0$ embeddings at fixed $T=0.1$ and show there is a second order transition. Note that the presence of this transition means the model has an intrinsic attractive interaction built into it-condensation would not occur otherwise. Below, we will investigate switching off this intrinsic attraction by switching on a repulsive four fermion interaction but also move to adjusting its strength to play the role of the QCD interactions.

The model has interesting structure beyond the basic transition. If in the broken phase we allow $\psi\left(r_{H}\right)$ to fall below the value that generates the $J_{c}=0, c \neq 0$ solution, there are solutions, shown in Fig. 1(b), that asymptote to negative $J_{c}$. A minimum $J_{c}$ is encountered as one lowers $\psi\left(r_{H}\right)$, and the UV value of $J_{c}$ then rises again. There is a further solution with $J_{c}=0, c \neq 0$, where the $\psi$ function dips once below the axis. This is an excited state of the vacuum where the first radially excited state of the bound states associated with $\psi$ has condensed rather than the ground state. As $\psi\left(r_{H}\right)$ falls, further excited states can occur, with condensation of higher and higher excitation modes. We demonstrate this by plotting the solutions in the $J_{c}, c$ plane for $\mu=5,10$ in Fig. 3, where a spiral structure is revealed. As the spiral moves between quadrants of the plane, the solutions for $\psi$ change-first, there are solutions for which $\psi$ is always positive, and then when the solution falls below the axis in the UV, we switch to negative $J_{c}$ and so on. This is typical in holographic models of symmetry breaking having first been identified in the D3/D7 system with a magnetic field [19]. These extra vacua will play an interesting role in the discussions to come.

We will next turn to introducing NJL interactions into the model. 


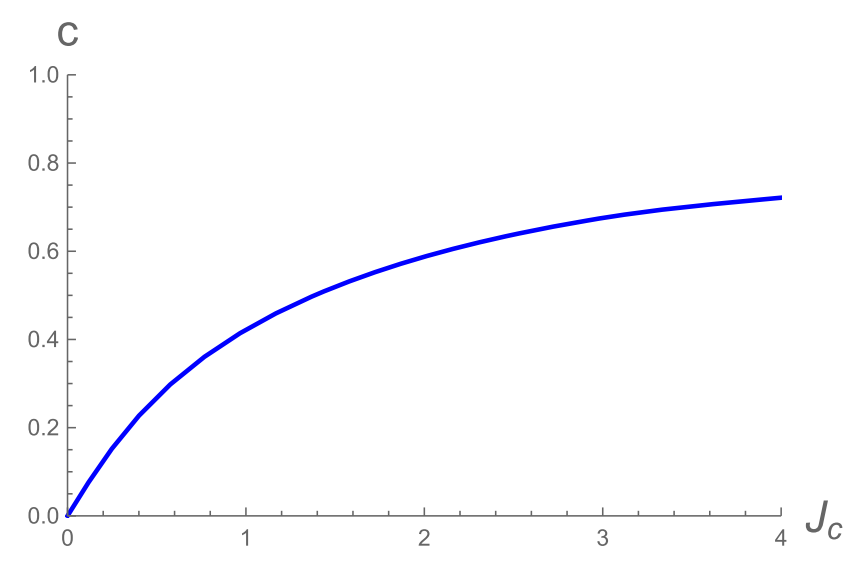

(a)

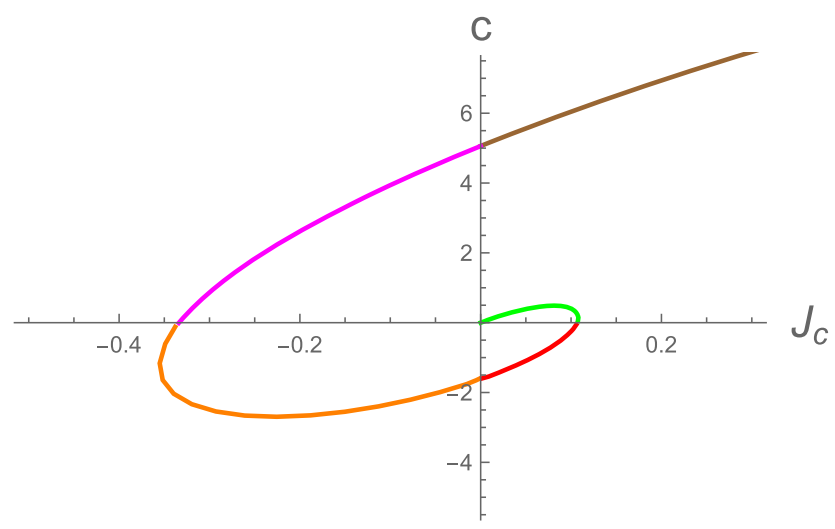

(b)

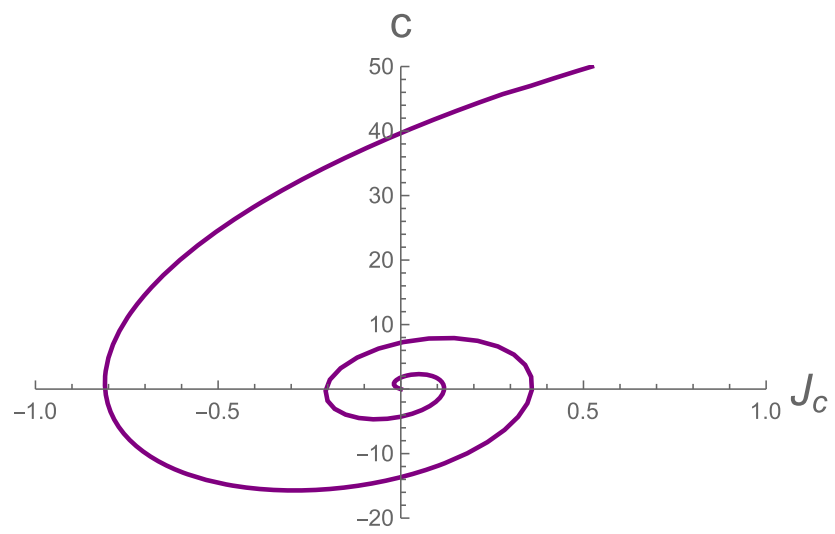

(c)

FIG. 3. $c$ vs $J_{c}$, where $T=0.1$. (a) Unbroken phase where $\mu=1.0$. (b): Broken phase where $\mu=5.0$. (c) Broken phase where $\mu=10.0$.

\section{NJL OPERATORS}

A natural step is to include the QCD interactions in our model of CSC as four fermion operators since the gluons are assumed to have acquired a large mass. Four fermion operators are an example of a "double trace" operator and can be incorporated using Witten's prescription [14]. Previous work on NJL operators in holographic superconductors can be found in Ref. [15], and recent work understanding the holographic description of the relativistic Nambu-JonaLasinio (NJL) model is in Ref. [16].

Consider the holographic description of an operator/ source pair $\mathcal{O}, J$ by a holographic field $\psi$ in $\mathrm{AdS}$,

$$
d s^{2}=r^{2} d x_{3+1}^{2}+\frac{d r^{2}}{r^{2}}
$$

with action (here, we pick $M^{2}=-3$ since all our operators are dimension 3)

$$
S=-\int d r \frac{1}{2}\left(r^{5}\left(\partial_{r} \psi\right)^{2}-3 r^{2} \psi^{2}\right) .
$$

The solutions take the form

$$
\psi=J / r+\mathcal{O} / r^{3} .
$$

Evaluating the action, there is a UV divergence, so we must include the counterterm at the UV boundary $(\Lambda)$ :

$$
\mathcal{L}_{\mathrm{UV}}=-\left.\frac{1}{2} \Lambda^{4} \psi^{2}\right|_{\Lambda}
$$

This term is crucial for the analysis below.

(Note that in the previous paper [16] we worked with a rescaled field $L=r \psi$. This is natural from the point of view of the D3/probe D7 system where the UV action takes precisely this form. If one substitutes this rescaled field into the action above and integrates by parts, then the surface term vanishes, and the action takes the form

$$
\mathcal{L}=-\int d r \frac{1}{2} r^{3}\left(\partial_{r} L\right)^{2},
$$

which, since $L \sim J+\cdots$, has no UV divergence and hence no counterterm. The IR boundary condition $\partial_{r} L=0$ forces $\mathcal{O}=0$, which is appropriate for supersymmetric gauge theory configurations where, e.g., the quark condensate is forbidden. Here, the action also vanishes with $L=$ constant corresponding to the vacuum energy of the gauge theory vanishing.)

We now wish to include in the field theory a term of the form

$$
\Delta \mathcal{L}=-\frac{g^{2}}{\Lambda^{2}} \mathcal{O O},
$$

where $\mathcal{O} \neq 0$; then, this term generates a source, $J=\frac{g^{2}}{\Lambda^{2}} \mathcal{O}$. If we substitute this relation back into the Lagrangian term, we uncover

$$
\Delta \mathcal{L}=-\frac{\Lambda^{2} J^{2}}{g^{2}}
$$

In analogy to this term, Witten's prescription in the holographic description is to add a UV surface term evaluated at the cutoff $\Lambda$, 


$$
\Delta \mathcal{L}=-\frac{\Lambda^{4} \psi^{2}}{g^{2}}
$$

since in the UV $\psi \sim J / \Lambda+\cdots$, one obtains (15).

The simplest way to include this extra term in the analysis is by considering the result of the change to the UV boundary condition on the solutions. Varying the action gives

$\delta S=0=-\int d r\left(\partial_{r} \frac{\partial \mathcal{L}}{\partial \psi^{\prime}}-\frac{\partial \psi}{\partial \psi}\right) \delta \psi+\left.\frac{\partial \mathcal{L}}{\partial \psi^{\prime}} \delta \psi\right|_{\mathrm{UV}, \mathrm{IR}}$.

There is also the variation of the surface counterterm

$$
\delta S=-\left.2 \Lambda^{4} \psi \delta \psi\right|_{\mathrm{UV}} .
$$

Normally, in the UV, one would require the source to be fixed and $\delta \psi=0$ to satisfy the boundary condition. We do this by fixing the source $J$ to specify a particular theory.

To describe the double trace operator, though, we allow $\psi(J)$ to change at the UV boundary and instead impose the vanishing at that boundary of

$$
0=\frac{\partial \mathcal{L}}{\partial \psi^{\prime}}+\psi \Lambda_{\mathrm{UV}}^{4}+\frac{2 \psi \Lambda_{\mathrm{UV}}^{4}}{g^{2}},
$$

where we have included the variation of the new surface term. For our action, $\frac{\partial \mathcal{L}}{\partial \psi^{\prime}}=-r^{5} \psi^{\prime}$. Assuming (11), we find that we need

$$
J \simeq \frac{g^{2}}{\Lambda^{2}} \mathcal{O}
$$

This condition [which matches the expectation under (14)] is simple to apply to the solutions of the (unchanged) equation of motion we already have.

\section{A. NJL operators in the superconductor}

Let us now return to the holographic superconductor model of the previous section. We can apply our analysis to the $\psi$ functions of Fig. 1. We can interpret each function, including those with $J_{c} \neq 0$, as describing the model with zero intrinsic $J_{c}$ but a four fermion operator present. The four fermion operator in the presence of the condensate $c$ generates the UV source $J_{c}$. For example, we can translate the functions of Fig. 1(a), where $\mu$ lies below the critical value, through Fig. 3(a), to a plot of $c$ against $g^{2}$, which we show in Fig. 4(a). Here, we have taken $\Lambda=10$ numerically. We observe a critical value of the NJL coupling that triggers symmetry breaking at a second order transition. Note here there are no solutions where in the $\mathrm{UV} J_{c}$ and $c$ have opposite signs-putting in a repulsive four fermion term (negative $g^{2}$ ) produces no solutions other than $J_{c}=0$, $c=0$ as one might expect.

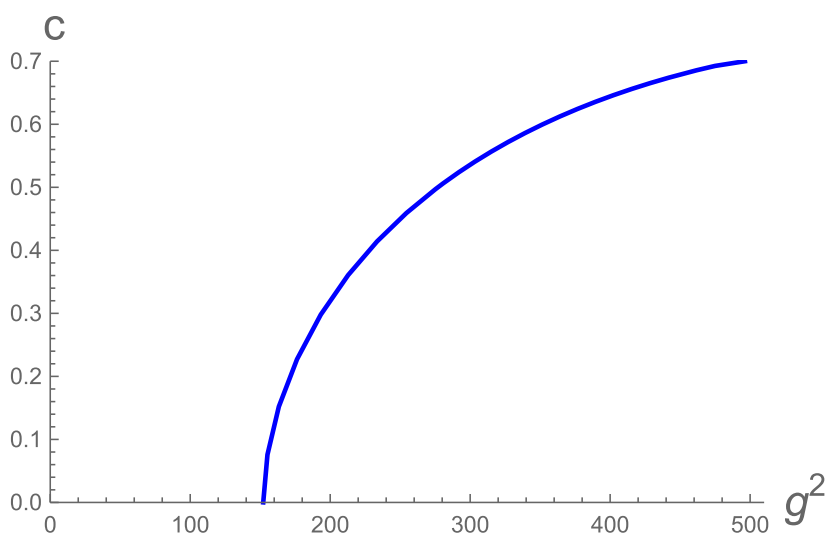

(a)

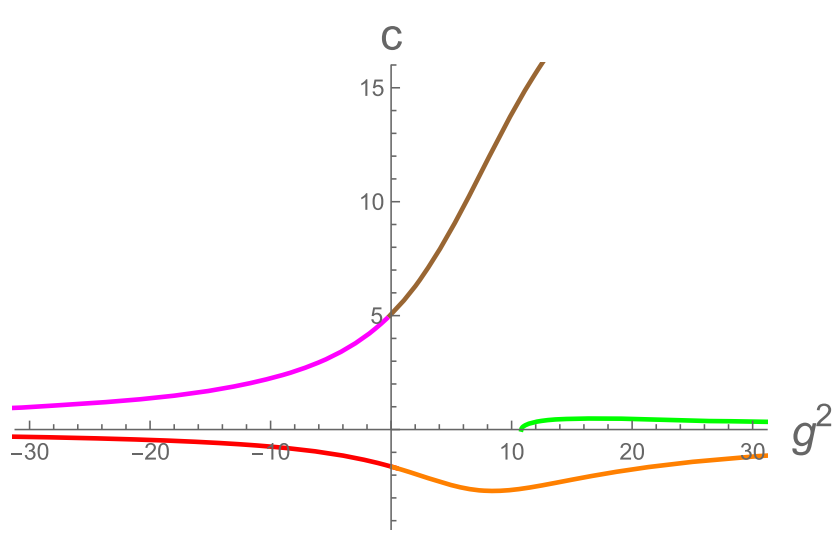

(b)

FIG. 4. (a) Plot of $c$ against $g^{2}(\Lambda=10)$ in the unbroken phase for embeddings in Fig 1(a) $(T=0.1, \mu=0.1)$. (b) Plot of $c$ against $g^{2}(\Lambda=10)$ in the broken phase $(T=0.1, \mu=5)$ for solutions in Fig. 1(b).

Similarly, we can translate the functions of Figs. 1(b)-3(b) to the plot in Fig. 4(b), which again shows $c$ vs $g^{2}$, but here at $g^{2}=0$, there is already symmetry breaking. There are two interesting additional features here. First, there are solutions at negative, repulsive, $g^{2}$. This is not surprising because at $g^{2}=0$ there is symmetry breaking-switching on a repulsive four fermion term would be expected to reduce the condensation, and it does. The surprising feature is that the condensation does not switch off completely except at infinite repulsive interaction strength (there are solutions with zero $c$ but nonzero $J_{c}$ that generate infinite $g^{2}$ values). The intrinsic attractive interaction in the AdS/superconductor model is presumably more subtle in structure than the NJL operator, which is only switching parts of the interaction off. Remember in superconductor theory any attractive interaction will result in condensation. The remaining structure in the $c-g^{2}$ plane is the translation of the spiral in the $c-J_{c}$ plane seen previously.

Our initial intention to describe QCD had been to take the basic holographic superconductor model and introduce a critically tuned repulsive NJL operator to switch off 


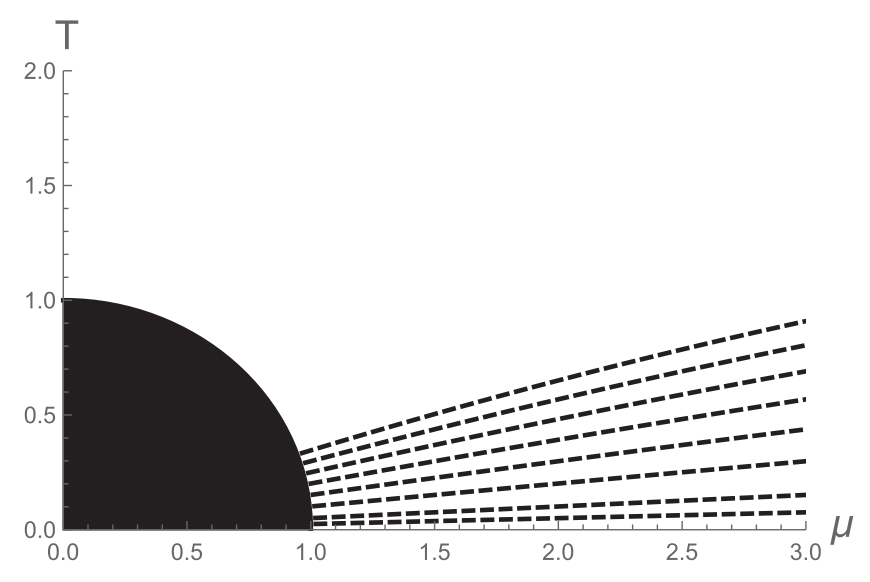

FIG. 5. Plot of the superconducting phase boundary at different $G=0.5,1,2,3,4,5,6,7$ from bottom to top in the $T$ - $\mu$ plane. The black region is expected to be the chirally symmetric phase below a scale of $\mu^{2}+T^{2}=1$.

condensation at each $T, \mu$ value. On this interaction free description of the quark gluon plasma, we would then add back the QCD interactions as further positive shifts in the NJL coupling strengths. We have now shown that this is not achievable because the intrinsic interactions are more subtle than the NJL interaction so an infinitely repulsive interaction is needed to switch off the base condensation. However, this approach was essentially to uninvent the wheel and then reinvent it. An equally sensible approach is to simply modify the strength of the interaction between $\psi$ and $A_{t}$ to reflect the QCD interaction strength. Our assumption is still that the gluons are massive in this strongly coupled phase so that we can describe the color of quarks by a global symmetry, but now the interactions will be introduced through the action

$$
\mathcal{L}=-\frac{1}{4} F^{\mu \nu} F_{\mu \nu}-|\partial \psi-i G B A \psi|^{2}+3 / L^{2} \psi^{2} .
$$

We interpret the $A \psi$ interaction term as the holographic models knowledge of the broken gauge interactions. Note the inclusion of the new coupling $G$, which we will shortly relate to the QCD running coupling.

First, though, we can find the phase boundary for the superconducting phase as a function of $G$. For each $T$ and $G$, we make plots as in Fig. 2 and then plot $\mu_{c}(T)$ in the plane. This is shown in Fig. 5. Note that, given the solutions for $G=1$, one can move to another $G$ by scaling $\psi \rightarrow G \psi$ and $A_{t} \rightarrow G A_{t}$ in (4) and (5) so the critical $\mu$ just scales with $G$.

\section{QCD PHASE DIAGRAM}

Let us now attempt to describe the color superconducting phase of QCD using these tools. We will assume that the chiral phase transition occurs at $T^{2}+\mu^{2}=\Lambda_{c}^{2}$ and numerically set $\Lambda_{c}=1$ with a UV cut off on the holographic model of $\Lambda=10 \Lambda_{c}$ where we read off $c, J_{c}$. We will assume a phase with a $\bar{q} q$ condensate lives below $\Lambda_{c}$.

In the quark gluon plasma phase, we will use the action of (21), but we must set the value of $G$ at the cutoff scale to a sensible ansatz in QCD. A natural choice based on the one loop running is

$$
G^{2}=\frac{\kappa}{b \ln \left(T^{2}+\mu^{2}\right) / \Lambda_{c}^{2}}, \quad b=11 N_{c} / 3-2 N_{f} / 3,
$$

which blows up at $\Lambda_{c}$. We need to fix $\kappa$ so it is appropriate for the strength of attraction that generates the $\overline{3}$ of color condensate.

Perturbatively, the strength of tree level t-channel one gluon exchange interaction for the four different color channels for $\bar{q} q$ and $q q$ is

$$
1_{\bar{q} q}: 8_{\bar{q} q}: 6_{q q}: \overline{3}_{q q}=-\frac{8}{3}: \frac{1}{3}: \frac{1}{6}:-\frac{1}{3} .
$$

The attraction might be as little as $1 / 8$, the attraction for the chiral condensate. Of course, at strong coupling, the relative strength of these interactions is not known.

The intrinsic interaction between the fields $\psi$ and $A_{t}$ in the holographic model should be controlled by the strength of the QCD interactions that presumably lie between $\kappa=1-(4 \pi)^{2}$. Given the $1 / 8$ th suppression, we will study the range of $\kappa$ between 1 and 20 to estimate the area of the phase diagram where superconductivity is likely.

It is now simple to construct the phase diagram from the analysis of Fig. 5. We overlay circles in the $T, \mu$ plane for each value of $G$ from (22) taking $N_{f}=3$ and identify the points where they cross the same $G$ value transition curve. We find the phase diagram in Fig. 6 . Very close to $\Lambda_{c}$, the coupling gets very strong, and the superconducting phase then hugs the phase boundary up to high values of T. Most likely, the chiral phase will extend a little above $\Lambda_{c}$, though,



FIG. 6. QCD phase diagram: the blacked out area is below $\Lambda_{c}$, where chiral symmetry breaking is expected. The remaining phase edges shows where the CFL phase is present for the choices of $\kappa=1,10,20$ from bottom to top. 
and this feature will be greatly reduced. Typically, we see the superconducting phase is predicted to exist below $\mathrm{T}$ of $0.15 \Lambda_{c}$ (for $\kappa \simeq 10$ ), which we might estimate as $20 \mathrm{MeV}$ or so if $\Lambda_{c} \simeq 175 \mathrm{MeV}$, the expected temperature of the chiral transition. This value might rise sharply just before the chiral transition. Usual estimates place the gap in the 10-100 MeV range [3], so this seems a sensible model.

\section{A. Quark mass}

Our phase diagram so far has been plotted for the massless theory, and the expected condensation is of the color-flavor locked form for $N_{f}=3$ and $2 \mathrm{SC}$ for $N_{f}=2$. One might expect there to be a transition as the strange quark mass grows from CFL to a two flavor 2SC phase at lower $\mu$. The presence of the mass leads to a lower value of the Fermi momentum, which will reduce the condensate, but also relative differences in the Fermi surface levels for different quark flavors are expected to frustrate the formation of the color-flavor locked condensate.

Holographically modeling this transition is not straightforward. Each component of (1) is of mixed flavor and should see the different chemical potentials and masses associated with each of the two constituents. Presumably this should be described by a non-Abelian Dirac-BornInfeld type action (assuming one could neglect the stringy nature of the states stretched between different flavor branes). Here, though, we will try something very naive to show a mechanism by which mass could switch off the condensation.

The quark mass, $m$, should be described by a new holographic field $\chi$ with asymptotic behavior $\chi=m / r+\cdots$ (the solution for a scalar of mass -3 in pure AdS) and IR dynamics that should be connected to the formation of the chiral condensate which is the subleading operator part of the solution. One would need a full model of the chiral transition to write down a potential for the $\chi$ scalar, so to avoid getting bogged down in that dynamics, we will just set $\chi=m / r$ and look at its effects on the $\psi$ Cooper pair formation (of course, really, one should solve linked equations, but our simplistic approach will show how the mass could suppress the Cooper pair condensation). We imagine a simple Lagrangian coupling of the form $|\chi|^{2}|\psi|^{2}$ so that the equation of motion for $\psi$ becomes

$\psi^{\prime \prime}+\left(\frac{f^{\prime}}{f}+\frac{5}{r}\right) \psi^{\prime}+\frac{G^{2} B^{2}}{r^{4} f^{2}} A_{t}^{2} \psi+\frac{1}{r^{2} f}\left(3-\frac{m^{2}}{r^{2}}\right) \psi=0$.

Clearly, the $m^{2}$ term acts to oppose the instability induced by $\mu$, which is the main mechanism we wish to flag here.

We first plot the phase boundary for $G=0.9$ at different values of $m$ in Fig. 7. As the quark mass rises, the boundary line tilts in the plane until for masses of order the chemical potential the phase is excluded at low $\mu$. The positive

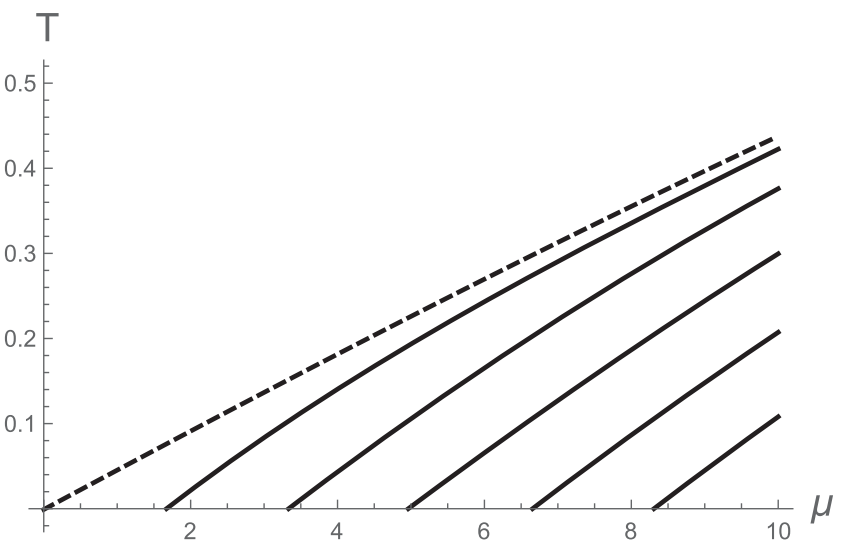

FIG. 7. Phase diagram for the model at fixed $G=0.9$ and for quark mass $m=0,1.3,2,3,4,5$ from left to right.

contribution to the scalar $\psi$ 's mass squared is greater than the BF bound violating negative contribution from $A_{t}$. The mass therefore discourages the condensation.

We plot the phase structure of the theory with the running coupling (22) for $\kappa=10$ and $m=0 ., 0.5,1.0$, 1.3 in Fig. 8. For small quark masses, the phase boundary simply moves to lower values of $T$ at a given $\mu$. If the mass becomes larger, though, then for a range of $\mu$, there is no condensation present. At large $\mu$, the mass is overwhelmed, and the condensation returns. Note at $\mu \simeq 1$ where the coupling becomes arbitrarily strong the phase briefly returns however large $m$ is, but this region is likely inside the chirally broken phase since the quark-antiquark attraction is also getting very strong.

In the case of QCD, one can interpret the above description as that for $\Delta_{1}$ and $\Delta_{2}$ with the interaction with $\chi$ describing the interplay with the strange quark mass. The phase boundaries in Fig. 8 for different $m$ represent our estimate of where the CFL phase $\left(\Delta_{1}\right.$ and $\left.\Delta_{2}\right)$ will switch off, although it is only a naive estimate since we have not

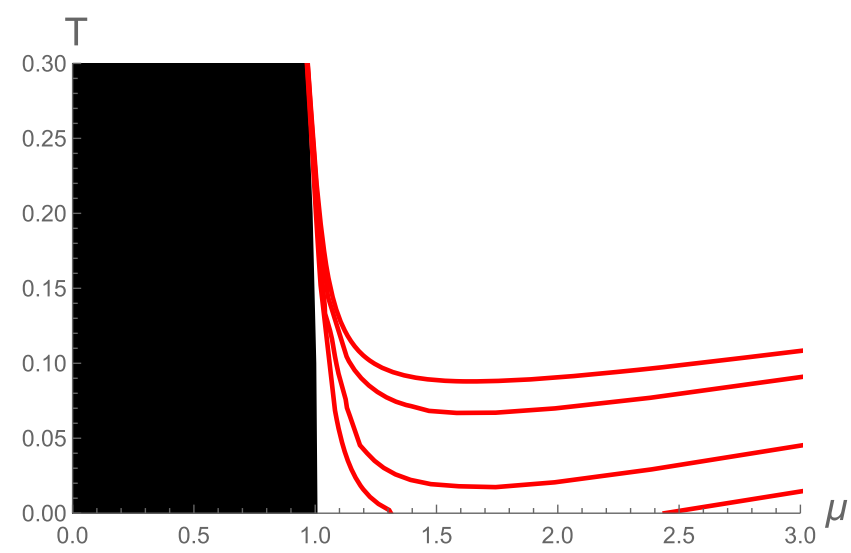

FIG. 8. QCD phase diagram with quark mass $m$ : in the blacked out area, chiral symmetry breaking is expected. The remaining phase edges show where the CFL phase is present for the choices of $\kappa=10$ and $m=0,0.5,1.0,1.3$ from top to bottom. 
included the effect of different Fermi surface levels. The 2SC phase would be expected to exist between the edge of the CFL phase and boundary for $m=0$ since $\Delta_{1}$ is oblivious to the strange mass. If we take $\kappa=10$ and assume $\Lambda_{c}=10 \mathrm{MeV}$, then the physical strange mass corresponds roughly to the $m=0.5$ curve, and the CFL phase exists down to the chiral boundary, although with a transition to the $2 \mathrm{SC}$ phase at higher T. For lower $\kappa$ values, the CFL phase might cease completely at lower $\mu$.

This discussion has been very naive, although it reveals a mechanism by which the CFL phase will be shut down by the strange quark mass, and we leave to future work including the correct dynamics for $\chi$, including the chiral transition, as well as incorporating the non-Abelian nature of the discussion.

\section{DISCUSSION}

The goal of this paper has been to push through the block in thinking as to how to describe the color superconducting phase of QCD holographically. For a long while, the color charged nature of the biquark condensate has stopped progress. We have attempted to side step this issue by arguing that at strong coupling and intermediate temperatures and chemical potentials the gluons are likely gapped by the plasma. If we treat the color symmetry of the quarks as a global index, then holographic models can progress. Indeed, here, we have demonstrated that by recycling the simplest holographic superconductor model adjusted to this setting. The key question was how to then include the QCD interactions. We investigated NJL operators as one possibility, and we have included the discussion here because there is an interesting story connected to the spiral structure in the operator-source plane in the superconductor model reflecting excited states of the vacuum (note that this structure is also present in the $\mathrm{AdS}_{4}$ superconductor but we are not aware of any discussion of it in the literature).
This leads to the conclusion that only an infinitely repulsive four fermion operator suffices to switch off the intrinsic attractive interaction of the holographic superconductor model. That attractive interaction is presumably more complex in structure than the four fermion operator, and any residual attraction would lead to superconductivity. In fact, we moved to simply adjusting the strength of that intrinsic interaction to reflect the QCD couplings value as a function of $\mu, T$. As a result, we can plot the phase diagram of the superconducting phase-see Fig. 6. The transition temperature lies near $20 \mathrm{MeV}$ or so, which matches the usually quoted range of $10-100 \mathrm{MeV}$.

The model we have used is somewhat like a NJL model of color superconductivity, but the holographic setting would allow one to easily compute equations of state and transport properties of the phases. We hope to investigate these and the consequences for neutron star structure and collisions in the future. There is also plenty of scope to make a more sophisticated model of the phase structure including backreaction on the metric, describing the chiral transition of QCD, and the interplay between the quark mass and the condensation pattern. We made a first attempt at understanding that mass dependence by a very simple model of an interaction between a quark mass and the Cooper pair which revealed a transition between a colorflavor locked phase and a 2SC phase-shown in Fig. 8. Again, this matches the form of the usually expected phase structure.

\section{ACKNOWLEDGMENTS}

N. E.'s work was supported by STFC consolidated Grant No. ST/P000711/1, and J.C.R.'s was supported by Mexico's National Council of Science and Technology Grant No. 439332. K. B. F. thanks B. Robinson for useful discussions and acknowledges the University of Southampton for its hospitality during his sabbatical.
[1] G. Benfatto and G. Gallavotti, J. Stat. Phys. 59, 541 (1990); Phys. Rev. C 42, 9967 (1990); R. Shankar, Physica (Amsterdam) 177A, 530 (1991); Rev. Mod. Phys. 66, 129 (1994); J. Polchinski, in Proceedings of the 1992 TASI, edited by J. Harvey and J. Polchinski (World Scientific, Singapore, 1993).

[2] N. J. Evans, S. D. H. Hsu, and M. Schwetz, Nucl. Phys. B551, 275 (1999).

[3] M. G. Alford, A. Schmitt, K. Rajagopal, and T. Schfer, Rev. Mod. Phys. 80, 1455 (2008).

[4] D. T. Son, Phys. Rev. D 59, 094019 (1999).

[5] J. M. Maldacena, Adv. Theor. Math. Phys. 2, 231 (1998); E. Witten, Adv. Theor. Math. Phys. 2, 253
(1998); S. S. Gubser, I. R. Klebanov, and A. M. Polyakov, Phys. Lett. B 428, 105 (1998).

[6] A. Karch and E. Katz, J. High Energy Phys. 06 (2002) 043; M. Grana and J. Polchinski, Phys. Rev. D 65, 126005 (2002); M. Bertolini, P. Di Vecchia, M. Frau, A. Lerda, and R. Marotta, Nucl. Phys. B621, 157 (2002); M. Kruczenski, D. Mateos, R. C. Myers, and D. J. Winters, J. High Energy Phys. 07 (2003) 049; J. Erdmenger, N. Evans, I. Kirsch, and E. Threlfall, Eur. Phys. J. A 35, 81 (2008).

[7] J. Erlich, E. Katz, D. T. Son, and M. A. Stephanov, Phys. Rev. Lett. 95, 261602 (2005); L. Da Rold and A. Pomarol, Nucl. Phys. B721, 79 (2005). 
[8] D. V. Deryagin, D. Yu. Grigoriev, and V. A. Rubakov, Int. J. Mod. Phys. A 07, 659 (1992); E. Shuster and D. T. Son, Nucl. Phys. B573, 434 (2000).

[9] N. J. Evans and M. Petrini, J. High Energy Phys. 11 (2001) 043.

[10] S. A. Hartnoll, C. P. Herzog, and G. T. Horowitz, Phys. Rev. Lett. 101, 031601 (2008).

[11] R. Apreda, J. Erdmenger, N. Evans, and Z. Guralnik, Phys. Rev. D 71, 126002 (2005); H. Y. Chen, K. Hashimoto, and S. Matsuura, J. High Energy Phys. 02 (2010) 104; A. F. Faedo, D. Mateos, C. Pantelidou, and J. Tarrio, J. High Energy Phys. 10 (2017) 139.

[12] A. Ramamurti, E. Shuryak, and I. Zahed, Phys. Rev. D 97, 114028 (2018).
[13] B. A. Freedman and L. D. McLerran, Phys. Rev. D 16, 1130 (1977); 16, 1147 (1977); 16, 1169 (1977).

[14] E. Witten, Multitrace operators, boundary conditions, and AdS/CFT correspondence, arXiv:hep-th/0112258.

[15] T. Faulkner, G. T. Horowitz, and M. M. Roberts, J. High Energy Phys. 04 (2011) 051.

[16] N. Evans and K. Y. Kim, Phys. Rev. D 93, 066002 (2016).

[17] M. G. Alford, K. Rajagopal, and F. Wilczek, Nucl. Phys. B537, 443 (1999).

[18] P. Breitenlohner and D. Z. Freedman, Ann. Phys. (N.Y.) 144, 249 (1982).

[19] V. G. Filev, C. V. Johnson, R. C. Rashkov, and K. S. Viswanathan, J. High Energy Phys. 10 (2007) 019. 